\title{
Hybrid, Endovascular, or Open Approach - the Paget Schroetter Syndrome Dilemma
}

\author{
Mohamad Bashir ${ }^{1}$, Cian Tan ${ }^{2}$, M Ghali Salahia ${ }^{3}$, Richard Whiston ${ }^{3}$, Richard White ${ }^{3}$, \\ Damian Bailey ${ }^{4}$, and Iam Williams ${ }^{3}$ \\ ${ }^{1}$ NHS Wales Health Education and Improvement Wales \\ ${ }^{2}$ Queen Mary University of London Barts and The London School of Medicine and \\ Dentistry \\ ${ }^{3}$ University Hospital of Wales \\ ${ }^{4}$ University of South Wales
}

July 7,2021

\begin{abstract}
Background Paget-Schroetter Syndrome (PSS) is an uncommon disorder involving thrombosis of the subclavian vein, often caused by repetitive overuse or compression by the surrounding anatomical structures. Optimal management of PSS is a subject of debate, but current trends suggest that a hybrid approach employing endovascular intervention and open decompression may yield the best clinical results. This original article examines the roles played by endovascular thrombolysis, surgical decompression, and postoperative secondary intervention in the management of PSS. Methods Current literature on the management of PSS was reviewed and evaluated to ascertain what strategy of intervention would be optimal. In addition, clinical data from the University Hospital of Wales on the clinical outcomes in PSS patients undergoing different surgical approaches for anatomical decompression are included. Results Evaluation of data from the included series and available literature seems to indicate that endovascular thrombolytic devices such as the AngioJet or mechanical thrombectomy offer superior results than traditional catheter-directed thrombolysis. In addition, adjunctive procedures such as superior vena cava filters and venous angioplasty or bypass may augment maintenance of the subclavian vein lumen. Nonetheless, the subclavian vein must still be relieved of pressure from surrounding structures for treatment to be successful. Conclusions A hybrid approach to the management of PSS, encompassing endovascular and surgical interventions could possibly offer optimal clinical outcomes as both intrinsic lesions and extrinsic compression of the subclavian vein are resolved. This article recommends prospective research to determine the ideal endovascular treatment, and best surgical approach for decompression.
\end{abstract}

\section{Journal of Cardiac Surgery - Wiley}

\section{Literature Review}

\section{Hybrid, Endovascular, or Open Approach - the Paget Schroetter Syndrome Dilemma}

Running head: Endovascular-Surgical Management of PSS

M Bashir MD, PhD, MRCS ${ }^{1}$, Sven ZCP Tan ${ }^{2}$, M Ghalia ${ }^{3}$, RJ Whiston FRCS ${ }^{4}$

RD White FRCR ${ }^{3}$, Damian M Bailey FRSC FPVRI FACSM FTPS ${ }^{5}$, IM Williams MD, FRCS ${ }^{4}$

1. Vascular and Endovascular Surgery, Health \& Education Improvement Wales, Wales, UK

2. Barts and The London School of Medicine and Dentistry, Queen Mary University of London, London, UK, E1 2AD

3. Department of Radiology, University Hospital of Wales, Health Park, Cardiff, Wales, UK, CF14 4XN 
4. Department of Vascular Surgery, University Hospital of Wales, Health Park, Cardiff, Wales, UK, CF14 $4 \mathrm{XN}$

5. Neurovascular Research Laboratory, Faculty of Life Sciences and Education, University of South Wales, Pontypridd, UK

Correspondence:

Mr IM Williams MD, FRCS

Regional Vascular Unit

Ward B2, University Hospital of Wales

Health Park, Cardiff, Wales, UK

CF14 4XW

$\mathrm{T}:+44(0) 2920747747$

F: $+44(0) 292074$

Email : Ian.Williams5@wales.nhs.uk

Funding declarations: D.M.B. is a Royal Society Wolfson Research Fellow (\#WM170007).

Conflicts of interest: M.B. is a Consultant for Terumo $\mathcal{E}$ a Consultant for JOTEC/CryoLife

Keywords: Paget-Schroetter Syndrome, Endovascular, Decompression, Thrombolysis,

\section{ABSTRACT}

\section{Background}

Paget-Schroetter Syndrome (PSS) is an uncommon disorder involving thrombosis of the subclavian vein, often caused by repetitive overuse or compression by the surrounding anatomical structures. Optimal management of PSS is a subject of debate, but current trends suggest that a hybrid approach employing endovascular intervention and open decompression may yield the best clinical results. This original article examines the roles played by endovascular thrombolysis, surgical decompression, and postoperative secondary intervention in the management of PSS.

\section{Methods}

Current literature on the management of PSS was reviewed and evaluated to ascertain what strategy of intervention would be optimal. In addition, clinical data from the University Hospital of Wales on the clinical outcomes in PSS patients undergoing different surgical approaches for anatomical decompression are included.

\section{Results}

Evaluation of data from the included series and available literature seems to indicate that endovascular thrombolytic devices such as the AngioJet or mechanical thrombectomy offer superior results than traditional catheter-directed thrombolysis. In addition, adjunctive procedures such as superior vena cava filters and venous angioplasty or bypass may augment maintenance of the subclavian vein lumen. Nonetheless, the subclavian vein must still be relieved of pressure from surrounding structures for treatment to be successful.

\section{Conclusions}

A hybrid approach to the management of PSS, encompassing endovascular and surgical interventions could possibly offer optimal clinical outcomes as both intrinsic lesions and extrinsic compression of the subclavian vein are resolved. This article recommends prospective research to determine the ideal endovascular treatment, and best surgical approach for decompression. 


\section{INTRODUCTION}

Paget-Schroetter syndrome (PSS) is a thrombosis of the axillary-subclavian vein (SV), due to repetitive use of the arm associated with 'the presence of one or more compressive elements in the thoracic outlet'. ${ }^{1}$ The condition mainly affects young males and is relatively uncommon for those with thoracic outlet syndrome, accounting for 1-4\% of all cases of venous thrombosis. The incidence of PSS is estimated to be between 1 to 2 per 100000 population. ${ }^{2}$ The SV is the most anterior structure traversing the first rib out of the thoracic cavity. The first rib is the most curved and usually the shortest of all the ribs and furthermore it is broad and flat. The head of the rib is small and rounded whilst the neck is narrow with the tubercle on the outer border. The upper surface has two shallow grooves with a ridge between them and is prominent medially as a bony prominence. This is the scalene tubercle where the scalenus anterior muscle inserts. The anterior groove is where the SV course whilst the subclavian artery and the lowest trunk of the brachial plexus runs in the posterior groove (Figure 1).

Apart from surrounding anatomical structures, intrinsic vascular factors exist that may dramatically increase the risk PSS. Chronic stenosis of the SV resulting from repeated motion-induced compression and paraneoplastic pro-thrombotic states are examples of documented intrinsic lesions that impede flow in the $\mathrm{SV}$ independent of surrounding structures, and lead to PSS. ${ }^{3}$

Current optimum management for PSS is immediate catheter-directed thrombolysis (CDTL) followed by surgical decompression of the SV. ${ }^{4}$ Such a hybrid approach would involve endovascular thrombolysis, perhaps via CDTL or mechanical thrombectomy, followed by open surgical decompression of local structures. These may then be followed by adjunctive angioplasty in acute settings or venous bypass in chronic cases. Proceeding with both endovascular and open surgical interventions ensures that intrinsic and extrinsic insults are dealt with, and could help to further mitigate the risk of recurrent thromboses - the combined approach has been noted to allow $100 \%$ of patients to regain preoperative function in the affected upper limb. ${ }^{4}$

Successful extravascular management of PSS depends on a thorough understanding of the anatomical structures that may impinge on SV and cause extrinsic compression. The subclavius muscle and tendon are located medially at the thoracic outlet and can potentially cause nutcracker-like compression of the SV with very minimal movement (Figures 1 and 2). The costoclavicular ligament inserts inferiorly to the upper medial aspect of the cartilage of the first rib and proceeds posterolaterally to the costal tuberosity on the inferior aspect of the medial clavicle. Rigberg in 2006 described the 'scissoring' effect between the clavicle and first rib with arm movement, to explain the potential for compression of neurovascular structures at the thoracic outlet. ${ }^{5}$

This review seeks to evaluate the roles of endovascular intervention against open surgical decompression for the management of PSS. Existing literature was reviewed to determine the significance of adopting both intervention modalities for the successful treatment of PSS. In addition, data gathered over 15 years in a tertiary referral centre in South Wales concerning the surgical approaches to anatomical resection are included. The outcomes scrutinised after thrombolysis, surgical decompression and venoplasty included vein patency, adjunctive measures performed at first admission and symptom relief.

\section{WHAT ROLE DOES ENDOVASCULAR INTERVENTION PLAY IN MANAGING PSS?}

Once the diagnosis of PSS is confirmed via history, physical exam, and imaging (such as X-ray of the thoracic inlet, duplex ultrasonography, or venography), holistic treatment typically proceeds in several stages, beginning with thrombolysis, followed by surgical decompression, and finally adjunctive angioplasty or venous bypass in certain cases. ${ }^{4}$

At this juncture, it is worth noting that a hybrid endovascular/open decompression approach is not always taken. Depending on local guidelines, patient characteristics, and myriad factors, the managing clinician may opt to proceed with only endovascular intervention or only open decompression. An approach employing both endovascular techniques alongside open decompression is associated with $100 \%$ technical success rates, and high rates of event-free survival. ${ }^{6}$ Zurkiya et al. emphasise that timely endovascular lysis or debulking 
of the thrombus is a well-documented contributing factor to the preservation of long-term luminal patency, and to the mitigation of re-occlusion, scarring, and intimal fibrosis. ${ }^{7}$ Further, Koury et al. suggests that surgical decompression may not be required if complete lysis is achieved via thrombolysis and there remains no evidence of residual stenosis or re-thrombosis. ${ }^{8}$

The endovascular management of PSS is chiefly centred on either CDTL or endovascular thrombectomy. Koury et al. describe the surgical technique for CDTL in upper extremity DVT (UEDVT) as beginning with gaining venous access in the ipsilateral upper extremity. ${ }^{8}$ Venography is carried out to determine the extent of thrombosis. The guidewire is then crossed over the lesion, to allow the infusion catheter to be introduced as near the lesion as possible. Thrombolytic agents such as tissue plasminogen activator or urokinase is then infused for a duration of at least 8 hours, or in some cases up to 72 hours. Patient fibrinogen levels and subclavian vein patency are monitored continuously. Catheter-related thrombosis can be prevented via infusion of a subtherapeutic dose of heparin. Koury et al. further note that CDTL is often augmented with mechanical thrombectomy, angioplasty, or endovascular stent placement to correct factors such as underlying stenosis. $^{8}$

Intriguingly, Landry et al. note that CDTL is often associated with prolonged treatment durations and adverse events including cerebral haemorrhage, pulmonary embolism, and access site bleeding. As a result, mechanical thrombectomy using devices such as the AngioJet used with the power pulse spray technique are being increasingly employed to tackle PSS (as opposed to iliofemoral DVTs). ${ }^{4}$

The AngioJet utilises a perforated catheter tip through which high-velocity, high-pressure streams of saline are projected. The resulting local region of low pressure allows entrapment and retrieval of bulky thrombi via the Venturi-Bernoulli effect. ${ }^{9,10}$ Though this system has historically been used more extensively for thrombus debulking in acute coronary syndromes and iliofemoral DVTs, Schneider and colleagues suggest the use of the AngioJet system in the setting of PSS is promising - they report an average treatment time of 12 hours overall, a significant reduction in comparison to that of CDTL. ${ }^{11}$

Completion of thrombolysis via endovascular intervention is then typically followed by surgical decompression of surrounding anatomical structures, before further adjunctive endovascular measures are implemented. Aggressive angioplasty prior to surgical decompression is generally discouraged, to prevent inflicting barotrauma to the wall of the SV while under anatomical compression.

\section{SURGICAL RESOLUTION OF EXTRINSIC VENOUS COMPRESSION}

Should thoracic outlet decompression be performed, historically, this has usually been via the transaxillary (TA) or supraclavicular (SC) approach. The SC approach provides excellent access for surgical decompression of the brachial plexus and subclavian artery but maybe difficult to access the SV due to its location anterior to the scalene muscles and posterior to the manubrium. For this reason, a paraclavicular (PC) or an infraclavicular (IC) incision has been considered an alternative option for successful surgical management of PSS. $^{2}$

\section{Surgical Approaches}

\section{TA Approach - Figure 3}

This requires meticulous preparation with the patient prepped to enable access via a $10 \mathrm{~cm}$ horizontal skin incision over the third rib. The lateral aspect of the pectoralis major and anterior border of the latissimus dorsi should be exposed. This incision exposes the fascia of the serratus anterior enabling the dissection to proceed proximally towards the apex of the axilla. Upward traction on the arm at this stage enables the scalenus anterior to be identified with the SV and artery either side. The insertion of the scalenus anterior into the first rib can then be divided exposing the anterior aspect of the first rib with the subclavius muscle visible under the head of the clavicle. This also needs to be divided at its origin from the medial aspect of the first rib taking care not to damage the SV. Inferiorly, the intercostal muscles are divided from the lateral aspect of the first rib which enables the pleura to be dissected free from the inferior aspect of the rib. The first 
rib can then be grasped and divided at the costochondral junction and divided as far posteriorly as necessary to isolate the SV and divide any extrinsic bands. The critical technical steps for successful decompression by the TA approach are excision of the anteriorly placed subclavius muscle and circumferential periadventitial dissection of the SV.

\section{IC Approach - Figure 4}

The incision commences $2 \mathrm{~cm}$ below the clavicle and extends medially to the sternal border. The two heads of the pectoralis major are identified and access between the clavicular and manubrial insertions. The advantages of the IC approach are principally a direct incision over the first rib in order to access the anteriorly located SV. Removal of this part of the first rib is crucial in the satisfactory treatment of SV thrombosis. This enables rigorous debulking of the costchondral junction and subclavius muscle resection from the origin at the superior aspect of the medial first rib. The intercostal muscles can be divided from the lateral rounded aspect of the first rib and medially the dissection can proceed posteriorly to divide the insertion of the scalenus anterior muscle. This incision should only be used when the SV needs the be decompressed as access to the artery and plexus are better served via the TA or with a separate SC incision. The IC incision can be combined with a SC approach - the PC. This enables more complete first rib resection than can be through the other individual approaches. Furthermore, as the SV is directly exposed, reconstruction can be performed as well.

\section{Postoperative management}

The final stage in management of PSS varies considerably. Patients often commence antiplatelet and anticoagulation therapy, with agents including aspirin and rivaroxaban respectively. Follow-up investigations include doppler ultrasound and venography to determine venous luminal patency in terms of blood flow at the lesion site, and to detect any residual stenosis or re-thrombosis that may require reintervention. ${ }^{12}$

Because thoracic outlet decompression in PSS fails to address intrinsic vessel wall lesions that may arise secondary to chronic anatomical compression, residual SV lesions that cause stenosis or recurrent thrombosis remain a particularly distinct cause for reintervention. ${ }^{3}$ In cases where persistent venous stenosis is detected on postoperative imaging, adjunctive percutaneous transluminal angioplasty (PTA) or stenting is a welldocumented approach to improve long-term clinical outcomes and maintain the re-establishment of the native luminal diameter. ${ }^{4}$ Schneider et al. note that up to $60 \%$ of patients persistently exhibit recurrent thrombosis or SV stenosis following surgical decompression, and that adjunctive angioplasty in this setting was found to be highly effective. Indeed, fibroelastic venous wall lesions in patients with PSS may necessitate balloon inflation pressures exceeding 10 atmospheres (atm) to achieve successful venous dilation. ${ }^{3,} 11$

Re-thrombosis in the SV lumen often occurs in the interval between decompressive surgery and adjunctive intervention, especially in cases where preoperative endovascular thrombolysis was not carried out, or full thrombolysis was not achieved. This has led surgeons to advocate for performing endovascular thrombolysis, decompression, and adjunctive procedures within a single hospitalisation. ${ }^{3}$ This approach would arguably speed up recovery time, and shorten the overall duration of admission and treatment. As an alternative, Koury et al. suggest prophylactic SVC filter placement prior to decompressive surgery, in cases where endovascular or systemic thrombolysis has failed or is contraindicated, especially when there is a backdrop of thromboembolic risk. Placement of such a filter at the confluence of the left and right innominate vein would therefore protect against cerebral, azygous, or pulmonary embolisation. ${ }^{8}$

It must be stressed, however, that adjunctive angioplasty or endovascular stenting does not serve to replace decompressive surgery -Schneider highlights that PTA in PSS patients who have not undergone decompressive surgery is especially ineffective. ${ }^{11}$ Though lumen patency may temporarily be maintained by the stent, anatomical compression during movement easily results in stent fracture and re-thrombosis of the SV. ${ }^{3}$

Adjunctive stenting is less effective in patients with chronic PSS because well-established thrombi typically respond poorly to balloon dilation as well as endovascular thrombolysis. In such instances, venous bypass using grafts from the saphenous or femoral veins, or indeed internal jugular turndown, may be carried out. ${ }^{4}$ 


\section{HOW DOES THE SURGICAL APPROACH TO DECOMPRESSION AFFECT CLINICAL OUTCOME?}

The following are derived from a retrospective review of PSS decompressive surgery at the University Hospital of Wales for the purpose of evaluation against hybrid treatment approaches (involving endovascular intervention, decompression, and adjunct therapy) for PSS.

\section{Methods}

Two vascular surgeons (IW/RJW) who routinely perform thoracic outlet surgery at University Hospital of Wales were included in the series. All patients presenting with a suspected diagnosis of PSS underwent complete history and examination followed by X-Ray of the thoracic inlet and duplex ultrasonography to confirm the diagnosis. If a thrombus was detected in the SV and there were no contraindications, a full discussion was held with the patient regarding venography and possible thrombolysis. The risks of thrombolysis were explained as were the potential benefits and if consenting underwent open surgical decompression following lysis. The selection of patients to undergo surgical decompression takes into account several factors. These include the age, whether it is the dominant arm affected, the duration of the thrombus within the vein, acceptance of risks of lysis and open surgery. Furthermore, if treated medically there is a risk of developing post phlebitic limb and associated morbidity in up to $46 \%$ of cases. ${ }^{13}$ Patients were initially treated with CDTL using tPA via the ipsilateral cephalic vein, at $1 \mathrm{~mL} / \mathrm{hr}$ after a $10 \mathrm{mg}$ bolus dose. This was repeated after 6 hours with a further venogram performed within 24 hours. tPA was either continued (for 6 further hours) or discontinued, depending on the residual thrombus load within the SV. Surgical decompression was then carried out in accordance with the IC, TA, and SC routes outlined previously, and all patients underwent first rib resection (FRR) under general anaesthetic. Patients were then commenced on antiplatelet therapy (aspirin) combined with 3-6 months of anticoagulation and latterly rivaroxaban. This was then stopped and antiplatelets were continued long-term. Venography was performed at six weeks post-surgery to assess patency and to treat residual stenosis if present.

\section{Results (Table 1)}

Twenty-six underwent surgical decompression for venous obstruction over a fifteen-year time period (2005 - 2020) and Eleven (42\%) were female with 15 (58\%) male. Median range for length of time of symptoms was 4 (2-30) days whilst length of time of TPA lysis was 22 (6-46) hours. One patient had a free floating first rib and three others had bony abnormalities related to the medial clavicle and costoclavicular junction (figures 5,6,7\&8). Three (12\%) had no intraluminal clot on initial Duplex ultrasonography and did not receive thrombolysis. Of the $23(88 \%)$ receiving lysis all underwent decompression surgically. Twelve (46\%) underwent surgery by the IC approach with $9(35 \%)$ by the TA route. Four (15\%) underwent a PC approach and one by the SC route. Of the 23 who underwent lysis, $16(72 \%)$ had a tight stenosis in the SV at the medial aspect of the first rib. Furthermore, 2 of these stenoses were greater than $2 \mathrm{~cm}$ in length. A further 3 were found to have a moderate SV stenosis after lysis and the remaining one an occlusion which was resistant to dilatation. Eleven venoplasties of the SV were performed - 5 prior to surgical decompression and 6 post. Follow up was for a median (range) of 36 (7-168) months and all 26 remained asymptomatic clinically with no evidence of post thrombotic sequelae in the affected arm.

\section{Outcomes}

Three SVs occluded post surgery (1 TA, 1 PC and $1 \mathrm{IC}$ ) at 36 months, 4 months and 1 day post decompression. Of these, 2 (TA and PC) underwent venoplasty which was successful in the first patient but in the second the venous occlusion was unable to be crossed with a wire. This was treated conservatively as was the patient occluding after one day post IC decompression. All three remained asymptomatic. Hence the secondary patency rates of SV were $23 / 26(88 \%)$ with all (100\%) asymptomatic.

\section{Symptom resolution}

Twenty-three had a patent SV on follow up. This included a patient who reoccluded after thirty six months and presented with a symptomatic upper limb venous thrombosis. This was treated with lysis (no venoplasty) 
and remains patent on anticoagulants. Two remain patent but with scarred post thrombotic SVs and the remaining two have chronically occluded SVs but remain asymptomatic following decompression.

\section{DISCUSSION}

Optimum surgical treatment of PSS arguably requires both endovascular intervention and adequate surgical decompression of the SV surrounding extrinsic structures. This is often further augmented via postdecompression secondary endovascular intervention or vascular reconstruction to ensure long term patency of the venous wall and lumen. Endovascular treatments such as mechanical thrombectomy performed in conjunction with CDTL, when combined with decompression and reconstruction as needed, seems to be particularly promising.

The clinical data from the series included in this literature review has shown that most patients undergoing thrombolysis and decompression had a definite venous wall stenosis secondary to the longstanding extrinsic compression: $42.3 \%(n=11)$ underwent venoplasty at some point. The durability of open surgery is impressive where adequate venolysis or early venoplasty seems to maintain patency of the SV and reduce rates of recurrent stenosis. Three developed SV occlusion post surgery of which one occurred on the first post operative day (symptomatic) and the second on routine venography four months later (asymptomatic). The third developed symptoms of arm swelling and pain thirty-six months post initial TA decompression which was lysed and underwent a further venoplasty successfully. The majority of patients in the series also had underlying SV stenosis following thrombolysis and decompression - possibly due to longstanding extrinsic compression of the SV leading to transmural fibrosis and ultimately thrombosis.

It must be highlighted that all patients in the cohort underwent thrombolysis via CDTL only. As outlined previously, CDTL is associated with several clinical limitations, such prolonged treatment time and key adverse events including pulmonary and cerebral embolism and entry site bleeding. ${ }^{4}$ Our review of current literature suggests that there exist viable alternatives to CDTL for the management of PSS. Schneider et al reported an average thrombolysis time of 12 hours overall (compared to 22 hours in the included series) when the AngioJet mechanical thrombolysis system was used for thrombus debulking prior to CDTL. ${ }^{11}$ Similarly, Shah et al. reported that use of the AngioJet system yielded successful thrombolysis in 2-3 hours for three patients. ${ }^{14}$ Results from Hileman et al. also seem to suggest the viability of mechanical thrombectomy as an alternative to CDTL in PSS: $93 \%$ of patients included had more than a $50 \%$ clot reduction when treated with mechanical thrombectomy, compared to $79 \%$ of patients treated with CDTL only. ${ }^{15}$ Furthermore, O'Sullivan and colleagues reported an average thrombolysis time of 91 minutes in using the Trellis thrombolysis catheter. ${ }^{16}$ This alternative to traditional CDTL, also conventionally used for treating LEDVT, was associated with $50-95 \%$ clot removal in $82 \%$ of patients, and $>95 \%$ removal in 3 patients. It was also associated with no major complications. ${ }^{16}$ The extent to which the residual SV stenosis experienced by the patients in the included series can be attributed to CDTL being performed over other thrombolysis methods is unclear. Though more research into the use of such endovascular devices would be prudent, it seems that approaches typically used to tackle lower-limb DVTs offer a promising step forward in the endovascular management of PSS.

Data from Wooster et al. also seems to suggest that endovascular intervention can help improve overall clinical outcome in patients with upper extremity DVT in general. Sixty seven percent of patients in their cohort underwent endovascular intervention, which included procedures such as PTA, patch PTA, and stenting. A $100 \%$ surgical success rate with symptomatic relief was observed in patients that underwent endovascular intervention in conjunction with decompression. Moreover, this approach was associated with low rates of SV reocclusion and symptom recurrence (9.4\% and $11.3 \%$ respectively). ${ }^{6}$ The clinical outcomes reviewed from available literature are summarised in Table 2.

Other contributory factors for re-thrombosis were incomplete resection of the costoclavicular ligament (CCL) and/or tendon of the subclavius muscle. The CCL is a rigid structure medial to the SV which can be seen by the TA and PC routes and causes SV compression particularly when the insertion into clavicle is lateral (figure 2). Similarly, the anteriorly lying subclavius muscle may be a further source of external 
compression on the SV and is easily identified by the IC route requiring excision to expose the vein. Recent series comparing the SC to the IC route for SV decompression have shown improved patency rates with fewer postsurgery symptoms for the IC approach ${ }^{17}$. Additionally, Molina et al. emphasise the importance of surgical access to the medial aspect of SV to adequately decompress the vessel surgically. ${ }^{18-21}$ The importance of carrying out decompression conjunction with endovascular interventions is therefore clear - as emphasised by Zurkiya et al., the degree of SV stenosis immediately post-decompression is usually comparable to that of pre-decompression because anatomical resection does not deal with intrinsic lesions to the SV. ${ }^{7}$ This also suggests that prolonging the interval between thrombolysis and decompression, and indeed between decompression and adjunctive intervention, would prove only detrimental to clinical outcome: time from presentation to decompression $>14$ days is a documented factor associated with worse clinical outcome, as it may lead to early re-thrombosis warranting re-intervention down the line. ${ }^{22}$

There is little current data concerning the approach surgeons use to manage PSS, both in terms of open decompression and endovascular intervention. A survey of 60 United Kingdom (UK) members of the Vascular Surgical Society showed $4 \%$ performed surgery for PSS by a PC incision with $55 \%$ opting for a TA approach and $28 \%$ for $\mathrm{SC}^{23}$. Hence, at least in the UK, there appears to be considerable variation in approaches adopted. This report dates to 2004 and clinical practice may have significantly changed since then.

Not a single randomised controlled trial has been conducted to determine the optimum surgical approach for PSS, perhaps due to its low incidence. Hence, it may not be feasible for a prospective randomised trial to be performed with sufficient power to gain a definitive answer as to even the optimum surgical approach to treat acute PSS - particularly if the TA and PC/IC outcome differences are minimal. What seems increasing clear, however, are the added benefits of undertaking endovascular intervention alongside surgical decompression, both in terms of preoperative thrombolysis and postoperative adjunctive venous repair.

A prospective randomised trial investigating the relative efficacies of the various approaches to preoperative endovascular intervention, for example mechanical thrombectomy and CDTL techniques, would therefore be prudent. Such a trial would help to elucidate whether the management of PSS, and indeed UEDVT in general, would benefit from treatment strategies traditionally employed to tackle LEDVT or even intraarterial pathologies. Indeed, the shift towards endovascular approaches has gained great popularity amongst surgeons managing complex cardiac and aortic pathologies - one needs only to look towards the advent of thoracic endovascular aortic repair as an example of the potential for endovascular procedures to successfully resolve complex cardiovascular surgical pathologies, without the need for large incisions and surgical trauma. ${ }^{24}$

\section{CONCLUSION}

Despite the distinct lack of prospective, large-scale multi-centre data on the optimal management of PSS, existing literature indicates that a hybrid approach encompassing endovascular intervention and surgical decompression yields respectable results that can be further augmented by post-decompression reconstructive procedures such as PTA and venous bypass. Methods such as mechanical thrombectomy coupled with traditional CDTL are particularly promising potential approaches and established thromboembolic complications of PSS could potentially be avoided with the fitting of SVC filters. Excellent long term SV patency rates are observed with both TA and PC/IC decompression approaches in the patients included in our series. Both approaches allow excellent access for complete resection of the anterior first rib, subclavius muscle and CCL eliminating any extrinsic compression precipitating the initial thrombotic event. Overall secondary patency rate was $92 \%$ with $100 \%$ asymptomatic. Post-surgical venoplasty and venous bypass are useful procedures which aid patency rates post decompression. At present evidence is lacking as to which patients may benefit from venous reconstruction but the IC approach enables this to be performed as medial access is easily obtained for control.

\section{References}

Urschel HC Jr, Kourlis H Jr. Thoracic outlet syndrome: a 50-year experience at Baylor University Medical Center. Proc (Bayl Univ Med Cent) 2007; 20:125-35. 
Illig KA, Doyle AJ. A comprehensive review of Paget-Schroetter syndrome. J Vasc Surg 2010; 51:1538-47.

Schneider DB, Dimuzio PJ, Martin ND, Gordon RL, Wilson MW, Laberge JM, Kerlan RK, Eichler CM, Messina LM. Combination treatment of venous thoracic outlet syndrome: open surgical decompression and intraoperative angioplasty. Journal of vascular surgery. 2004 Oct 1;40(4):599-603.

Landry GJ, Liem TK. Endovascular management of Paget-Schroetter syndrome. Vascular. 2007 Oct $1 ; 15(5): 290-6$.

Rigberg DA, Freischlag JA, Machleder HI. Vascular compression syndromes. In: Creager MA, Dzau V, Loscalzo J, editors. Vascular medicine: a companion to Braunwald's heart disease. 1st ed. Philadelphia: Elsevier; 2006. p. 920-33.

Wooster M, Fernandez B, Summers KL, Illig KA. Surgical and endovascular central venous reconstruction combined with thoracic outlet decompression in highly symptomatic patients. Journal of Vascular Surgery: Venous and Lymphatic Disorders. 2019 Jan 1;7(1):106-12.

Zurkiya O, Donahue DM, Walker TG, Ganguli S. Safety and efficacy of catheter-directed therapies as a supplement to surgical decompression in venous thoracic outlet syndrome. American Journal of Roentgenology. 2018 Feb;210(2):W80-5.

Koury JP, Burke CT. Endovascular management of acute upper extremity deep venous thrombosis and the use of superior vena cava filters. InSeminars in interventional radiology 2011 Mar (Vol. 28, No. 1, p. 3). Thieme Medical Publishers.

Lee MS, Singh V, Wilentz JR, Makkar RR. AngioJet thrombectomy. Journal of Invasive Cardiology. 2004 Oct 1;16:587-95.

Villalba L, Nguyen T, Feitosa Jr RL, Gunanayagam P, Anning N, Dwight K. Single-session catheter-directed lysis using adjunctive power-pulse spray with AngioJet for the treatment of acute massive and submassive pulmonary embolism. Journal of vascular surgery. 2019 Dec 1;70(6):1920-6.

Schneider DB, Curry TK, Eichler CM, Messina LM, Gordon RL, Kerlan RK. Percutaneous mechanical thrombectomy for the management of venous thoracic outlet syndrome. Journal of Endovascular Therapy. 2003 Apr;10(2):336-40.

Stewman C, Harwood M. Thoracic outlet syndrome [Internet]. BMJ Best Practice. 2020 [cited 27 June 2021]. Available from: https://bestpractice.bmj.com/topics/en-gb/592

Elmann EE \& Khan SR. The post-thrombotic syndrome after upper extremity deep venous thrombosis in adults: a systematic review. Thromb Res 2006;117(6):609-614.

Shah AD, Bajakian DR, Olin JW, Lookstein RA. Power-pulse spray thrombectomy for treatment of PagetSchroetter syndrome. American Journal of Roentgenology. 2007 May;188(5):1215-7. 
Hilleman DE, Razavi MK. Clinical and economic evaluation of the Trellis-8 infusion catheter for deep vein thrombosis. Journal of Vascular and Interventional Radiology. 2008 Mar 1;19(3):377-83.

O'Sullivan GJ, Lohan DG, Gough N, Cronin CG, Kee ST. Pharmacomechanical thrombectomy of acute deep vein thrombosis with the Trellis- 8 isolated thrombolysis catheter. Journal of vascular and interventional radiology. 2007 Jun 1;18(6):715-24.

Bozzay JD, Walker PF, Ronaldi AE, Patel JA, Koelling EE, White PW, Rasmussen TE, Golarz SR \& White JM. Infraclavicular thoracic outlet decompression compared to supraclavicular thoracic outlet decompression fir the management of venous thoracic outlet syndrome. Ann Vasc Surg 2020;65:90-99.

Molina JE, Hunter DW, Dietz CA. Protocols for Paget-Schroetter Syndrome and late treatment of chronic subclavian vein obstruction. Ann Thorac Surg 2009; 87:416-22.

Molina JE. A new surgical approach to the innominate and subclavian vein. J Vasc Surg 1998; 27:576-81.

Molina JE. Reoperations after failed transaxillary first rib resection to treat Paget-Schroetter Syndrome patients. Ann Thorac Surg 2011; 91:1717-22.

Molina JE, Hunter DW, Dietz CA. Paget-Schroetter syndrome treated with thrombolytics and immediate surgery. J Vasc Surg 2007; 45:328-34.

Samoila G, Twine CP \& Williams IM. The infraclavicular approach for Paget-Schroetter syndrome. Ann Roy Coll Surg Engl 2018 feb:100(2);83-91.

Khan SN, Stansby G. Current management of Paget-Schroetter syndrome in the UK. Ann R Coll Surg Engl 2004; 86:29-34.

Harky A, Chan JS, Wong CH, Francis N, Grafton-Clarke C, Bashir M. Systematic review and meta-analysis of acute type B thoracic aortic dissection, open, or endovascular repair. Journal of vascular surgery. 2019 May 1;69(5):1599-609.

\section{Acknowledgements}

The authors wish to thank Melissa Melling for Figures 1-4.

\section{Hosted file}

Figure 1 PSS.docx available at https://authorea.com/users/340497/articles/529496-hybridendovascular-or-open-approach-the-paget-schroetter-syndrome-dilemma

\section{Hosted file}

Figure 2 PSS.docx available at https://authorea.com/users/340497/articles/529496-hybridendovascular-or-open-approach-the-paget-schroetter-syndrome-dilemma

\section{Hosted file}

Figure 3 PSS.docx available at https://authorea.com/users/340497/articles/529496-hybridendovascular-or-open-approach-the-paget-schroetter-syndrome-dilemma 


\section{Hosted file}

Figure 4 PSS.docx available at https://authorea.com/users/340497/articles/529496-hybridendovascular-or-open-approach-the-paget-schroetter-syndrome-dilemma

\section{Hosted file}

Figure 5a PSS.docx available at https://authorea.com/users/340497/articles/529496-hybridendovascular-or-open-approach-the-paget-schroetter-syndrome-dilemma

\section{Hosted file}

Figure 5b PSS.docx available at https://authorea.com/users/340497/articles/529496-hybridendovascular-or-open-approach-the-paget-schroetter-syndrome-dilemma

\section{Hosted file}

Figure 6a PSS.docx available at https://authorea.com/users/340497/articles/529496-hybridendovascular-or-open-approach-the-paget-schroetter-syndrome-dilemma

\section{Hosted file}

Figure 7a PSS.docx available at https://authorea.com/users/340497/articles/529496-hybridendovascular-or-open-approach-the-paget-schroetter-syndrome-dilemma

\section{Hosted file}

Figure 7b PSS.docx available at https://authorea.com/users/340497/articles/529496-hybridendovascular-or-open-approach-the-paget-schroetter-syndrome-dilemma

\section{Hosted file}

Figure 7c PSS.docx available at https://authorea.com/users/340497/articles/529496-hybridendovascular-or-open-approach-the-paget-schroetter-syndrome-dilemma

\section{Hosted file}

Figure 8a PSS.docx available at https://authorea.com/users/340497/articles/529496-hybridendovascular-or-open-approach-the-paget-schroetter-syndrome-dilemma

\section{Hosted file}

Figure 8b PSS.docx available at https://authorea.com/users/340497/articles/529496-hybridendovascular-or-open-approach-the-paget-schroetter-syndrome-dilemma

\section{Hosted file}

Table 1 PSS.docx available at https://authorea.com/users/340497/articles/529496-hybridendovascular-or-open-approach-the-paget-schroetter-syndrome-dilemma

\section{Hosted file}

Table 2 PSS.docx available at https://authorea.com/users/340497/articles/529496-hybridendovascular-or-open-approach-the-paget-schroetter-syndrome-dilemma 